\title{
Evience of episodic wind events in Deneb
}

\author{
R.E.M. Griffin \\ Herzberg Institute for Astrophysics, DAO, Canada
}

\begin{abstract}
We report irregular changes in the line profiles of Deneb, and suggest that they represent part of the mechanism causing the stellar wind.
\end{abstract}

Keywords. Stars: early-type, stars: supergiants, stars: indiviual: Deneb, stars: winds, outflows, line: profiles, techniques: spectroscopic

\section{Introduction}

High-dispersion, high $S / N$ spectra of Deneb ( $\alpha \mathrm{Cyg}$, A2 Ia), centred near $\lambda 4481$, were observed in March 2002 with the DAO 1.2-m telescope and high-dispersion ('9682') camera/grating coudé configuration, as part of a project concerned with late-B and early-A stars. I noticed some very slight differences in the line profiles compared to a different occasion. The differences were manifested as very weak, red-shifted components in the wings of all the prominent lines in that region of the spectrum. I then began to monitor the spectrum fairly frequently, several times during observing runs in $2002 / 3$, but not necessarily on each night. On most occasions I made two consecutive observations (usually around 7-8 minutes each) and coadded the spectra, acquiring a set of 25 single or averaged spectra with typical $S / N$ of $400-800$.

In April 2004 when I submitted this paper's abstract I had not intercompared all the spectra. I was still under the impression that I had detected extra absorption in the red line-wings. But my investigation of the symmetry of the lines now indicates that actually the converse is the case, those spectra were the "normal" ones, while on other occasions the red-wing profile is slightly filled in by emission.

One could also postulate that the observed lack of symmetry is caused by blue-shifted absorption in the blue wing. However, application of Occam's Razor suggests that the cases of symmetry are the norm against which the others are to be compared.

\section{Observational evidence}

To study the line asymmetries, I isolated Mg II $\lambda 4481$ and reflected it about its midpoint so that the blue and red wings were superimposed (see Fig. 1). Although $\lambda 4481$ is a close blend of two equal components (and can be resolved by the DAO spectrograph in the case of very narrow-lined stars), the natural line-broadening in Deneb is sufficient to render it effectively single.

Twelve spectra, classified as "symmetrical", were averaged to form a template. The template was then divided into each of the the others in turn, revealing residual emission/absorption wherever it occurred - see Fig. 2.

Figs. 3-5 are enlargements of parts of Fig. 2. The dotted lines are red-shifted by 35 $\mathrm{km} \mathrm{s}^{-1}$ from the rest wavelengths of the identified features. The lines all arise from the following ions: $\mathrm{Mg}$ II, Ti II and Fe II. 


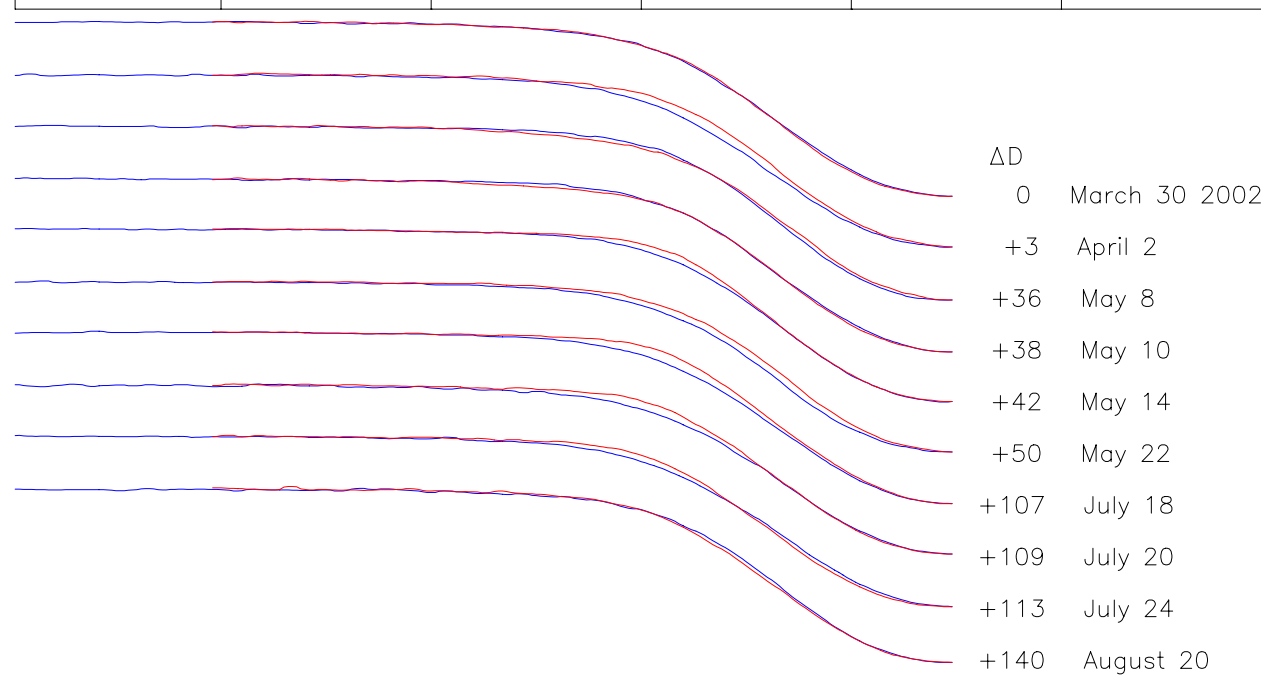

Figure 1. Line-profiles of $\mathrm{Mg}$ II $\lambda 4481$ with the red wing reflected onto the blue one. $\Delta \mathrm{D}$ is in days.

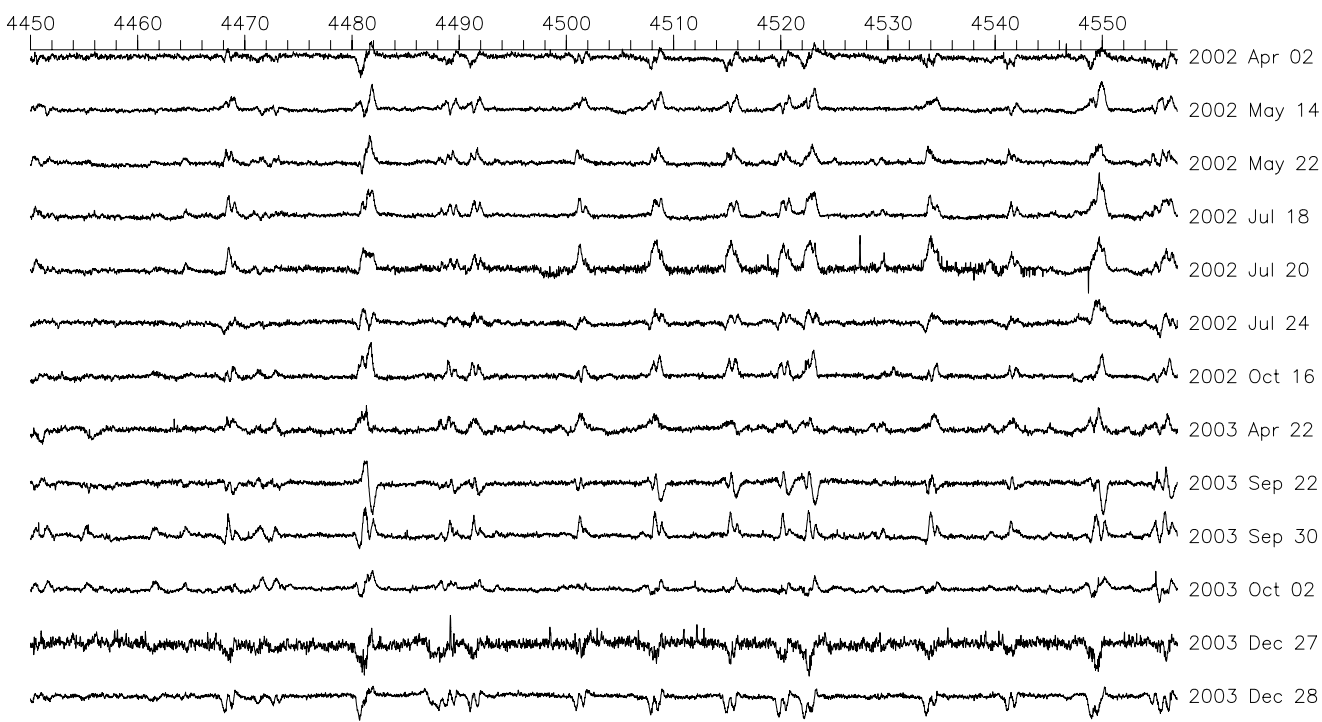

Figure 2. Residual features in spectra of Deneb after removing the symmetrical spectrum.

\subsection{Observational and processing errors}

We recall that slight differences in the purity of the original spectra (for instance, in the sharpness of the focus) can give rise to false "emission" or "absorption" in residual spectra. However, such artefacts are expected to be symmetrical about the positions of the unshifted lines.

Small errors in the wavelength scale will give rise to artificial P-Cyg profiles, which will occur randomly in either the usual sense or inverted, since the sign of the wavelength errors is random. 


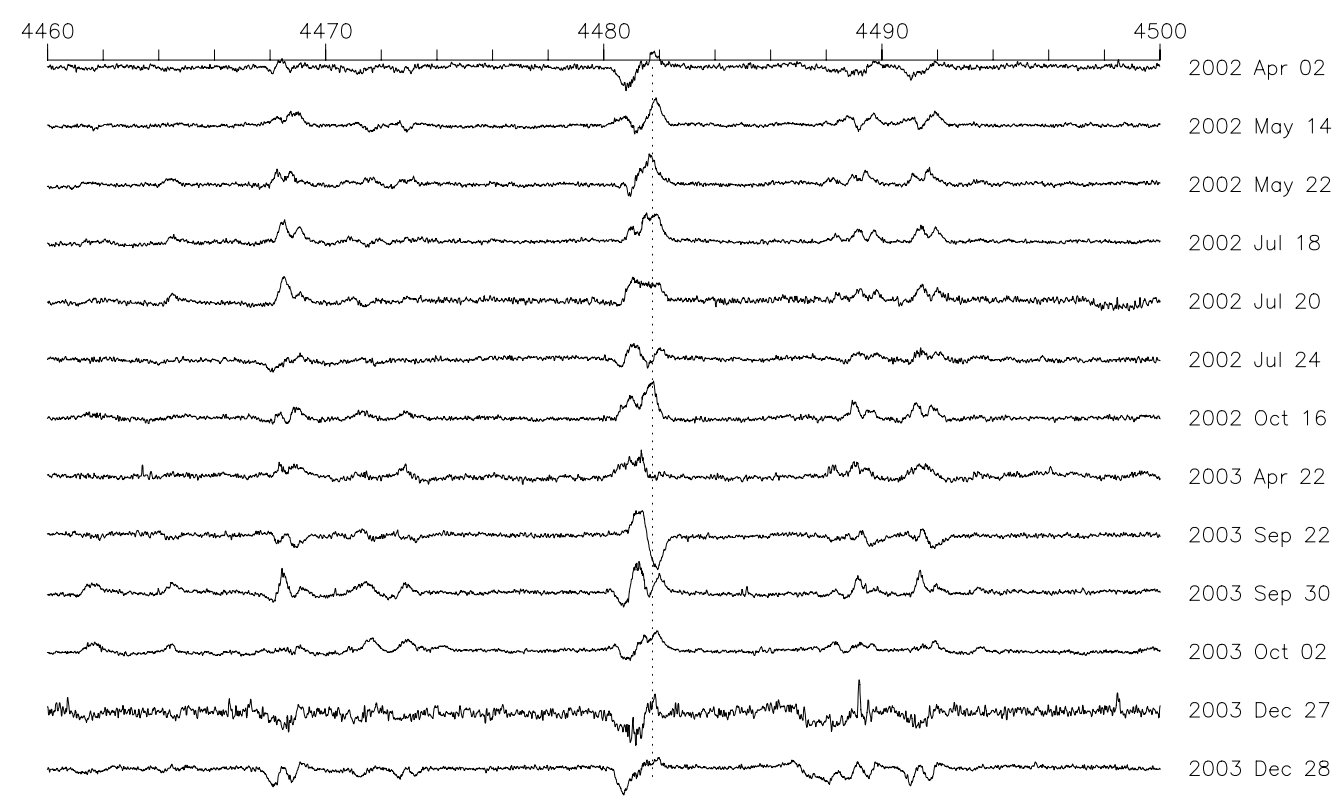

Figure 3. Sequence of residual profiles in Mg II $\lambda 4481$.

I determine the wavelength scales of my spectra in the rest-frame of the star, by identifying well-defined lines in the stellar spectrum and applying the grating equation. For Deneb I used the same set of identified lines throughout, and with the same spectrograph parameters modified only by small alterations in grating angle and in the temperaturedependent focal length of the camera. Since any wavelength-scale error will be small, the corresponding false P-Cyg features will occur close to the line core. However, the observed asymmetries occur well out in the wings, so one can be confident that they have not been caused by errors in wavelength scales. Moreover, by forming my template spectrum from an average of 12 spectra I will have reduced the random errors in its wavelength scale.

Furthermore, because the observed asymmetries are weak and occur well away from the line cores they will not have noticeably affected my measurements of the positions of the line centres.

\section{Winds and mass loss in A-type supergiants}

It has been known for at least 50 years that A-type supergiants show photometric and spectrum variabilities, exhibiting not only semi-periodic radial-velocity variations but also changes in the intensity and widths of line profiles, over time-scales of 5-50 days. The best catalogued line-profile changes in the visible have been observed at $\mathrm{H} \alpha$, which often shows a very broad emission, plus a red-shifted emission of between $50-100 \mathrm{~km} \mathrm{~s}^{-1}$ and often an almost symmetrical blue-shifted one as well.

Newer evidence from UV spectroscopy of Deneb, and more recently from millimetre and centimetre frequencies, indicates an admixture of a continuous outflow, with both broad and narrow discrete absorptions in resonance lines. These observations suggest a multi-structured stellar wind, with at least one component causing a "puff" or shell, with an ejection frequency of the order of one year (Talavera \& Gomez de Castro 1987).

Deneb manifests such activity, but at a rather modest level. The broad component is 


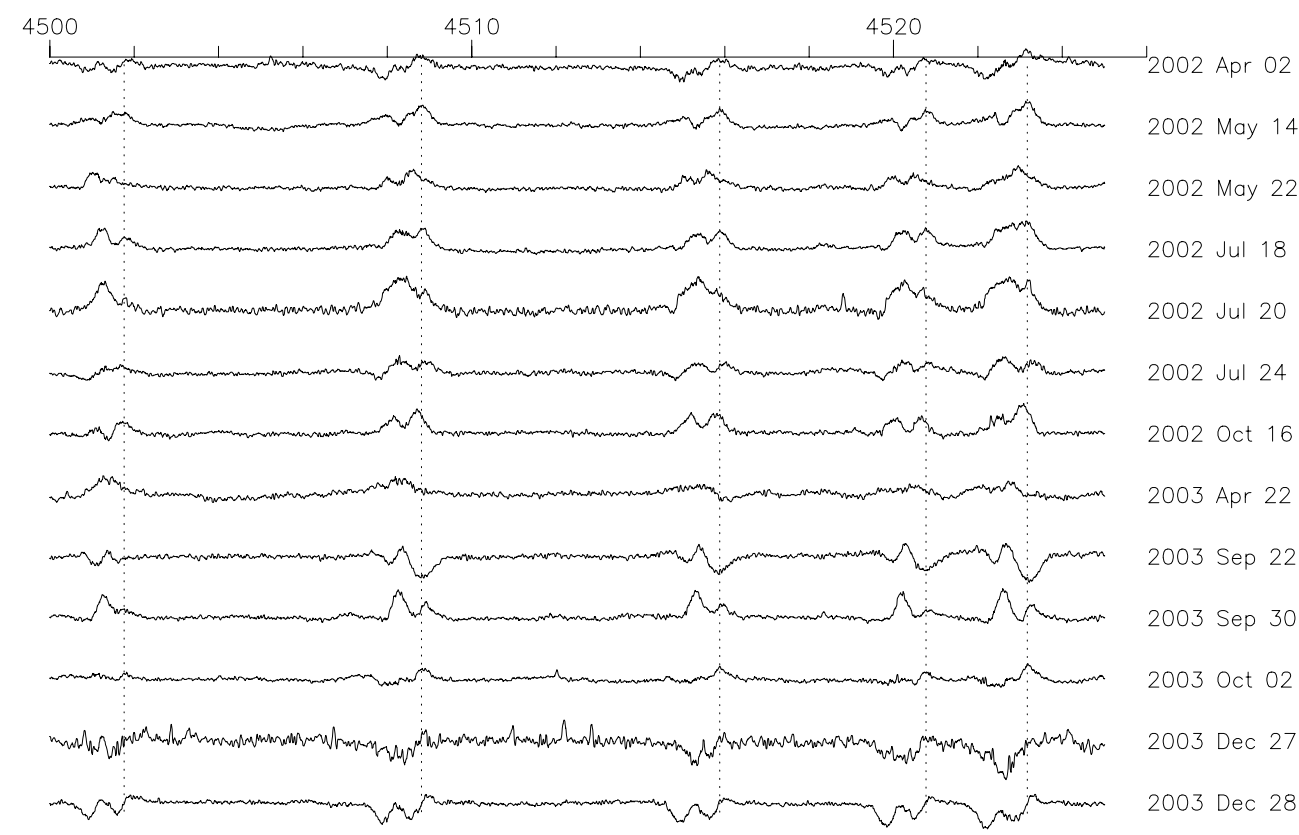

Figure 4. Sequence of residual profiles at $\lambda 4501$ (Ti II), and $\lambda 4508,4515,4520$ and 4523

(Fe II).

not seen, and the red-shifted emission is detected only insofar as it fills in some of the line absorption. However, the nature of the time-scales (of the order of days to months but not necessarily periodic) means that appropriate monitoring at high $S / N$ and high resolution on such a bright object as Deneb $(V=1.25)$ has been difficult to secure in competition with faint-object work.

A-type supergiants are important to extragalactic astronomy as potential distance indicators through application of the wind momentum-luminosity relationship derived from radiation-driven stellar wind theory. However, when such a model was derived for Deneb, by way of a test (Aufdenberg et al. 2002), the authors were unable to match the $\mathrm{H} \alpha$ profile at all satisfactorily. It is important to understand the reasons for the apparent failure, as it undermines the credibility of the method and its wider application. Even today the mass-loss rates for a star as well-observed as Deneb range over three orders of magnitude, depending on the type of observation in question and on the method of analysis, whether UV Mg II profiles, IR excess or radio flux limits. Any new observational evidence in this regard is therefore particularly valuable.

\section{Changes without periodicity}

Taken at face value, Fig. 2 demonstrates line-profile changes that are extensive, and cyclical without being strictly periodic. The pattern suggests a variable emission, mostly red-shifted by $30-40 \mathrm{~km} \mathrm{~s}^{-1}$ but occasionally less. There is also some evidence of doublepeaked emission, such as is seen clearly in the $\mathrm{H} \alpha$ monitoring by Kaufer et al. (1996) and even in the same sense, the redward of the two peaks usually being the stronger. Where that is clearly not the case (e.g., on 2003 September 30) the pattern is consistent for all the lines. However, whereas for $\mathrm{H} \alpha$ Kaufer et al. reported a red emission at $+20 \mathrm{~km} \mathrm{~s}^{-1}$ 


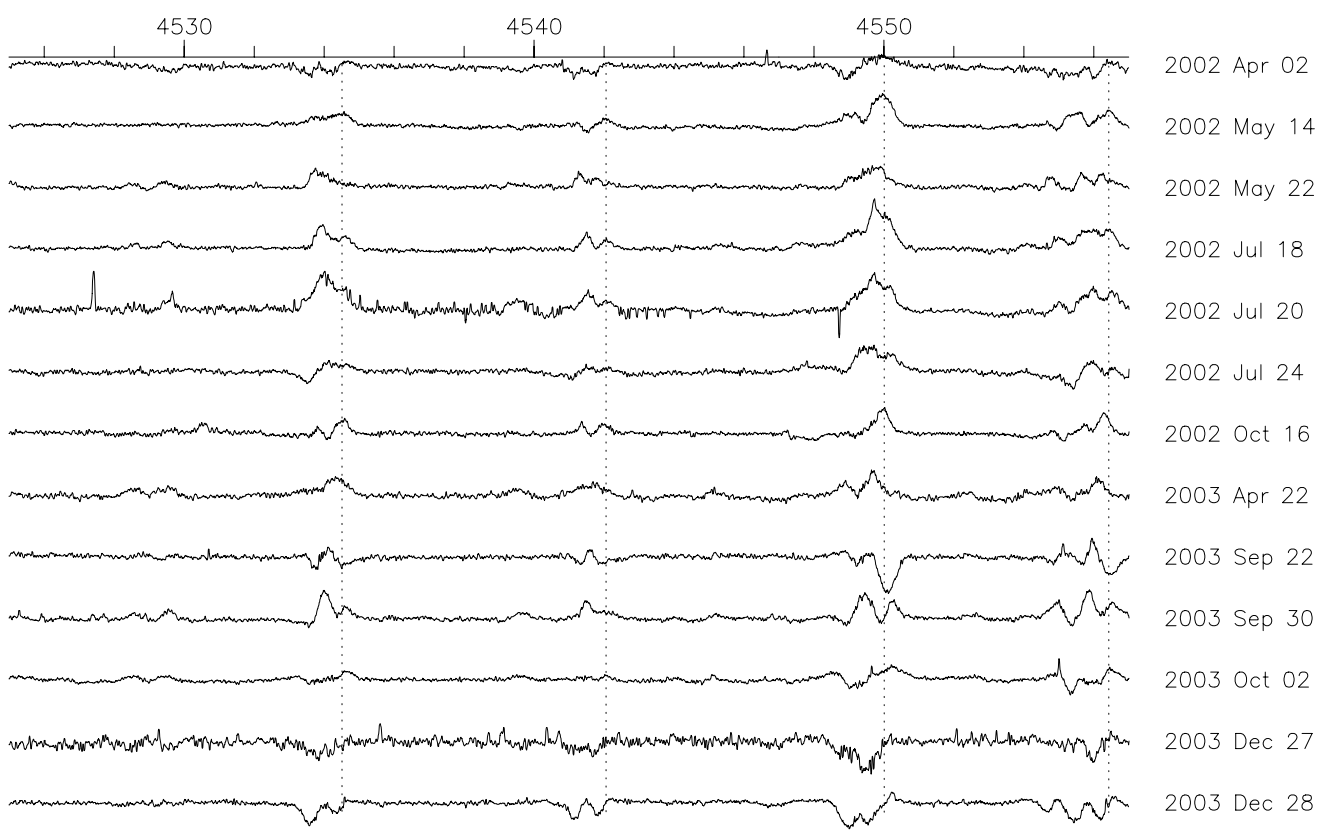

Figure 5. Sequence of residual profiles at $\lambda 4534$ ( $\mathrm{Ti}$ II), and $\lambda 4541,4549$ and 4556 (Fe II).

and a blue-shifted one near $-60 \mathrm{~km} \mathrm{~s}^{-1}$, the velocity displacement between the peaks in Figs. $3-5$ is only about half as great.

On 2002 May 22 and 2002 July 18 the profiles of the emission match quite well with respect to the detailed pattern, but the later set is more red-shifted by about $12 \mathrm{~km} \mathrm{~s}^{-1}$ (see Fig. 6). However, other aspects of Figs. 2-5 suggest that the characteristic time-scale is often a few days rather than two months.

\section{Additional evidence}

Some spectra of other regions of Deneb were also observed during 2002/3, 3 at $\mathrm{H} \delta$ and 11 centred at the $\mathrm{Ca}$ II $\mathrm{K}$ line but including $\mathrm{H} 8$ at $\lambda 3889$. When $\mathrm{H} \delta$ and $\mathrm{H} 8$ were divided by the respective spectra supposedly without emission, we uncovered red-shifted emission of a very similar nature to that found in Mg II $\lambda 4481$ (see Figs. 7 and 8). (The $\mathrm{K}$ line itself could not be used because of the $I S$ line near its core.)

Note: Because of ambiguity over the choices of both "symmetry" templates (the dates of observation were not the same as for the Mg II $\lambda 4481$ ), the results from Figs. 7 and 8 should be regarded as preliminary only.

\section{Interpreting the optical spectroscopy}

Although it is the blue-shifted absorptions which carry direct evidence of the stellar wind, the red-shifted emission components of the P-Cyg profiles are the easier to detect and measure reliably in these optical spectra. From the irregularity of those components we can deduce the occurrence of episodic events in the wind from Deneb, while from the behaviour of lines of different elements and in different Balmer lines we can postulate that they are part of the same mechanism revealed in $\mathrm{H} \alpha$ but occurring at a different mean heights. In the case of H8, Fig. 8 suggests an inward propagation of the emission, 

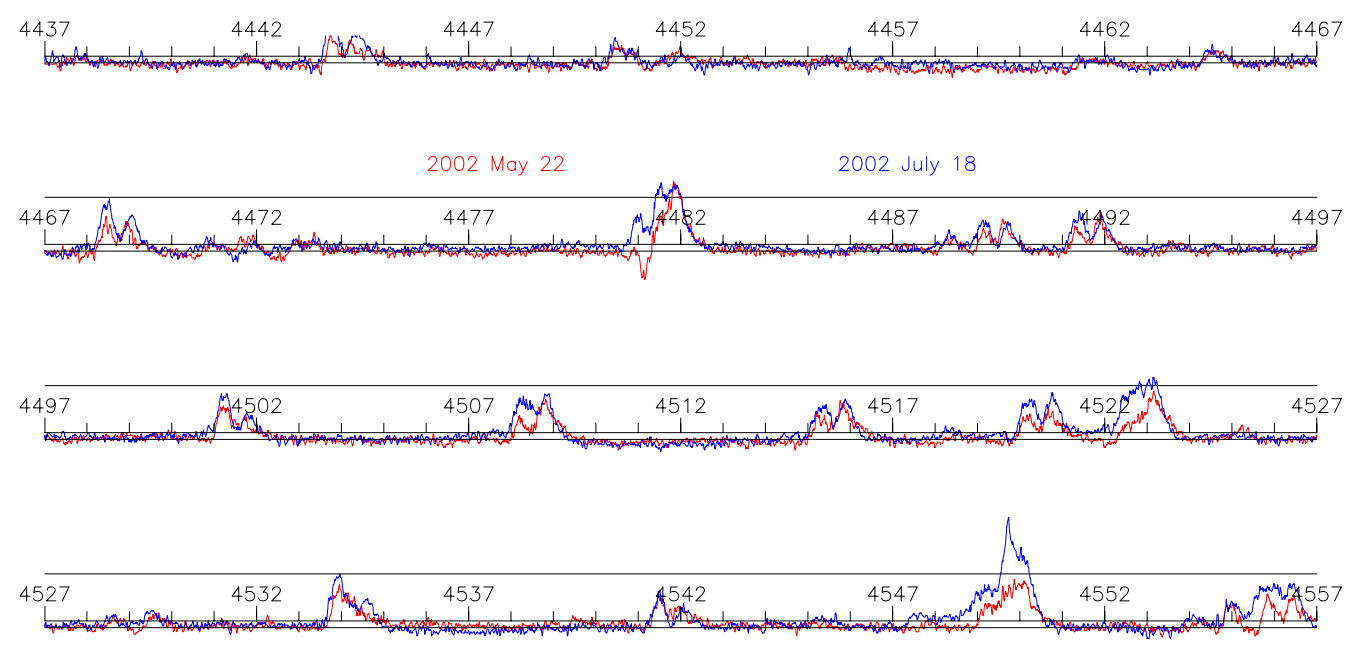

Figure 6. Emission profiles two months apart. The later one has been blue-shifted by $12 \mathrm{~km} \mathrm{~s}^{-1}$ to match the earlier one.

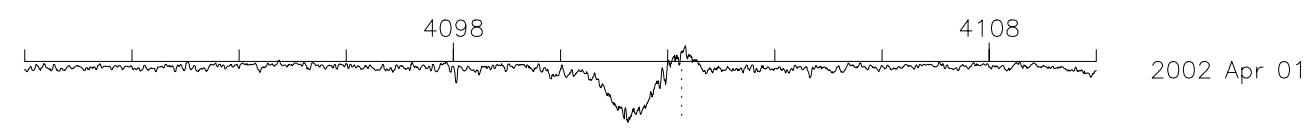

Figure 7. Emission at $+37 \mathrm{~km} \mathrm{~s}^{-1}$ in the profile of $\mathrm{H} \delta$.

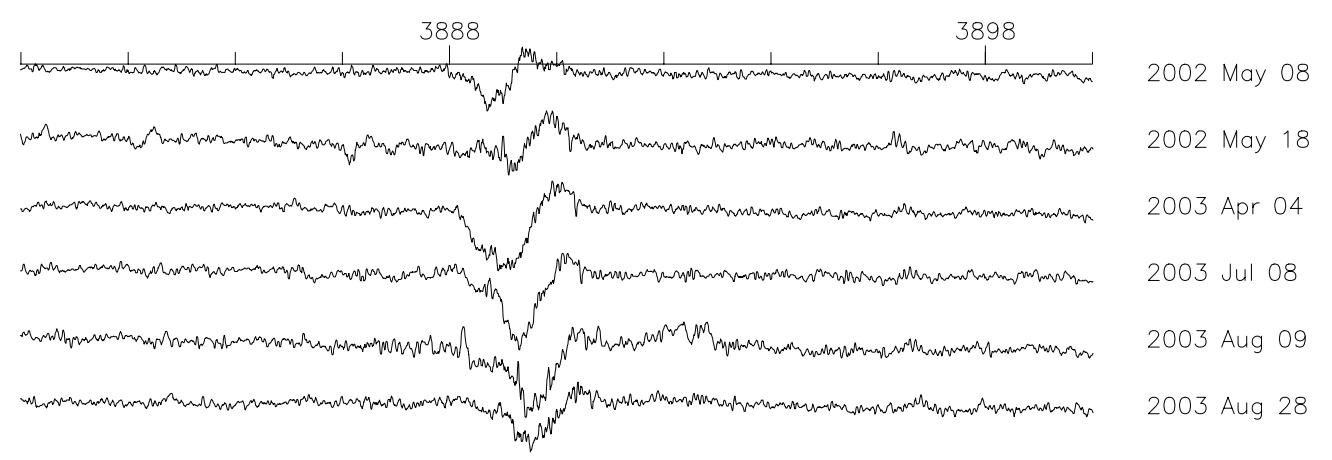

Figure 8. Emission at H8. The emission velocity increases from +27 to $+100 \mathrm{~km} \mathrm{~s}^{-1}$.

with a velocity increasing from about 30 to $100 \mathrm{~km} \mathrm{~s}^{-1}$. However, in two spectra observed in May 2003 the red-shifted emission was not present. The wind events thus appear to be not only irregular but rapid, and may correspond to the "puffs" first mentioned by Lamers et al. (1982).

Measurements of the time-scales involved will provide valuable input for modelling these supergiant A-star winds, but it is far from clear what constitutes a characteristic time-scale. Plots of the measured velocities of the red-most emission did not show any clear pattern, probably because the observations were too clustered and intermittent. We, 
therefore, plan to continue monitoring the spectrum of Deneb on a nightly basis, when possible, over a few weeks, to determine the shortest characteristic interval of change.

This paper illustrates once again the value of high-dispersion spectroscopy on bright stars. It also highlights (once again) how much there is still to be learned about even a relatively quiescent stellar atmosphere.

\section{Acknowledgements}

REMG is grateful to the DAO and to the Symposium organizers for financial assistance to attend this meeting.

\section{References}

Aufdenberg, J.P. et al. 2002, ApJ 570, 344

Kaufer, A. et al. 1996, A\&A 305, 887

Lamers, H.J.G.L.M., Gauthier R. \& Snow, T.P. 1982, ApJ 258, 186

Talavera, A., Gomez de Castro, A.I. 1987, A\&A 181, 300 